\title{
Circuit
}

Musiques contemporaines

\section{Création contemporaine et partage des traditions : à la rencontre de Katia Makdissi-Warren}

\section{Vicky Tremblay}

Volume 29, numéro 3, 2019

URI : https://id.erudit.org/iderudit/1066490ar

DOI : https://doi.org/10.7202/1066490ar

Aller au sommaire du numéro

Éditeur(s)

Circuit, musiques contemporaines

ISSN

1183-1693 (imprimé)

1488-9692 (numérique)

Découvrir la revue

Citer ce document

Tremblay, V. (2019). Création contemporaine et partage des traditions : à la rencontre de Katia Makdissi-Warren. Circuit, 29(3), 96-98.

https://doi.org/10.7202/1066490ar d'utilisation que vous pouvez consulter en ligne.

https://apropos.erudit.org/fr/usagers/politique-dutilisation/ 


\section{Création contemporaine et partage des traditions : à la rencontre de Katia Makdissi-Warren}

Vicky Tremblay

La Série hommage à Katia Makdissi-Warren présentée par la Société de musique contemporaine du Québec (SMcQ) est l'occasion de mettre de l'avant les musiques de cette compositrice au parcours et à la démarche uniques. Ses œuvres se singularisent par les rencontres entre diverses traditions (du MoyenOrient au chant de gorge inuit) et sont empreintes des différentes identités culturelles qui s'y retrouvent. En ce sens, le constat de Serge Provost quant à la situation des créateurs en musique depuis la fin du $\mathrm{xx}^{\mathrm{e}}$ siècle fait écho à la démarche de la compositrice :

De nos jours, les débats esthétiques ont largement supplanté le dogmatisme théorique et s'orientent vers des discussions qui touchent à la singularité des systèmes de référence et témoignent d'une profonde recherche d'identité de la part des créateurs ${ }^{1}$.

La recherche mentionnée par Provost se présente chez Makdissi-Warren par la rencontre de différentes identités culturelles qui se croisent au sein de son œuvre. Par le métissage, la compositrice parvient à redéfinir la musique contemporaine et contribue à son décloisonnement vers de nouvelles traditions. Ses années de travail avec l'ensemble Oktoécho illustrent cette redéfinition par les dialogues et les échanges avec les interprètes de l'ensemble, mais aussi par le respect de entretiens avec la compositrice ont permis un retour et des réflexions sur son parcours et sa démarche à la fois singulière et novatrice.

\section{Parcours et résistance}

C'est d'abord au Conservatoire de musique de Québec que Makdissi-Warren entreprend ses études en composition dans les années 1990. Sa démarche suscite une certaine résistance de la part des professeurs, ce qui ne l'empêche pas de poursuivre ses études ainsi que son projet de rencontre entre les musiques occidentales et moyen-orientales. Elle se rend par la suite en Italie et en Allemagne pour approfondir son étude de la création musicale auprès d'Ennio Morricone et de Manfred Stahnke, puis à Beyrouth, où elle s'imprègne des traditions de la musique arabe (populaire et classique) avec P. Louis Hage. «J'ai tenté d'intégrer ce que j’ai découvert au Liban dans tout ce que j’avais appris en étudiant la composition occidentale²», soulignet-elle. À cet égard, son doctorat en composition - réalisé à l'Université de Montréal sous la direction de Michel Longtin - est l'occasion de synthétiser la diversité de ses apprentissages au Québec, en Europe et au Moyen-Orient.

Si Makdissi-Warren assume pleinement son identité stylistique, son statut de compositrice de musique 
contemporaine n’a pas toujours été reconnu. Elle explique :

On sent beaucoup d'ouverture de la part des nouvelles générations. Il n’y a plus autant de barrières ou de «snobisme» musical. Mais ça n’a pas toujours été comme ça. Lorsque j’ai commencé à composer, il y avait de réelles barrières dans les universités et les conservatoires entre musique contemporaine, musique jazz, musique populaire, musique du monde, etc.

De fait, certains ont considéré d'emblée que son œuvre appartenait à la musique populaire. «On cherchait ainsi à dénigrer mon œuvre et la musique populaire à la fois! », déplore-t-elle. Si la compositrice soutient que plusieurs ont souffert de ces barrières imposées, elle ajoute que le désir de créer sans contraintes permet de dépasser ces tentatives d'étiquetage. Par ailleurs, «aller au-delà de ce qui était préconçu au Conservatoire permettait d'ouvrir à une source d'inspiration musicale infinie », se souvient-elle.

\section{Métissage et identités}

La musique de tradition classique est donc aux fondements de la démarche de création de Makdissi-Warren, et le métissage lui permet d'établir une rencontre signifiante avec d'autres traditions. Selon elle, l'identité et la rencontre sont deux pôles en interaction constante lorsqu'il est question de métissage: «Il faut toujours savoir qui nous sommes, nos origines, ce que nous voulons faire, et ce, en collectivité. » Ainsi, la compositrice affirme que c'est grâce à cet équilibre entre une identité musicale revendiquée et une rencontre signifiante qu'il est possible de trouver un point commun, un espace dans lequel les musiciens se retrouvent.

Selon elle, «il est impératif de ne pas dépasser les limites de l'étrangeté pour les interprètes. Si on dépasse ces limites, le processus d'interprétation n'a plus de sens pour eux. » En suivant ce principe, il est évident que la démarche de création de Makdissi-Warren se doit d'être flexible, parfois même adaptée aux inter- prètes de sa musique. Aussi n'écrira-t-elle pas de la même façon pour un ensemble comme Oktoécho ensemble pour lequel elle compose régulièrement et dont elle assure la direction artistique - que pour un orchestre symphonique.

\section{Oktoécho: un espace de partage des traditions}

En 2006, la compositrice crée Oktoécho, un projet réunissant des musiciens issus des traditions moyenorientales et occidentales. Si ses œuvres sont interprétées par des formations de tradition classique telles que l'Orchestre symphonique de Québec, l'Ensemble Contemporain de Montréal ou l'Orchestre de chambre de l'Université McGill, Oktoécho s'inscrit en tant qu'espace de création essentiel pour Makdissi-Warren. Les musiciens peuvent y interpréter ses œuvres métissées sans pour autant « dépasser leur étrangeté».

Dans Oktoécho, en effet, les interprètes tiennent un rôle important dans le processus de création:

Avec Oktoécho, j’ai le luxe de savoir pour qui j’écris. Je compose différemment selon les forces de la personne pour qui j'écris. Parfois, j'écris très peu, car je sais que l'interprète ajoutera exactement ce qu'il faut.

Un autre aspect important du travail d'Oktoécho est le temps. Prendre le temps de se rencontrer, d'échanger. Par exemple, dans Saimaniq (le projet de rencontre avec les chanteuses de gorge inuites), le processus de création s'est amorcé par des ateliers:

Nous avons commencé par environ vingt heures d'ateliers. Nous avons improvisé, essayé des mélanges entre les différents instruments, etc. À la fin des répétitions, j'enregistrais le résultat et je retravaillais des partitions à partir du travail que nous avions réalisé.

Ce processus de création a mené à l'enregistrement d'un album, paru en $2017^{3}$. Les ateliers ont pourtant commencé en 2011: "J'aime bien prendre le temps que tout "mûrisse". De plus, nous avons développé à travers ces années de forts liens de confiance. » 
Cette confiance ainsi qu'un immense respect ressortent de façon significative dans l'œuvre d'Oktoécho et de Katia Makdissi-Warren. Ils permettent un réel partage entre les différentes traditions: une rencontre qui sort des perspectives eurologiques ${ }^{4}$ (s'inscrivant uniquement dans la tradition classique occidentale de la musique sans considérer réellement les procédés d'interprétation et de création des autres traditions) et qui mène vers un décloisonnement des frontières entre la musique contemporaine et les différentes traditions, ces dernières étant si essentielles au développement d'une société véritablement inclusives.

\section{B I B LI O G RAPHIE}

Bhagwati, Sandeep (2018), «Glossaire raisonné », Circuit, musiques contemporaines, vol. $28, \mathrm{n}^{\circ} 1$, p. 15-22.

MaKdissi-Warren, Katia (2007), «La rencontre des musiques du Moyen-Orient et de l'Occident: esthétisme composition et analyse ", thèse de doctorat, Université de Montréal.
Provost, Serge (2003), "Complexité/simplicité/complexité », in Jean-Jacques Nattiez (dir.), Musiques: une encyclopédie pour le $\mathrm{XxI}^{e}$ siècle. Vol. 1: musiques du $\mathrm{Xx}^{e}$ siècle, Paris, Actes Sud/Cité de la musique, p. 620-631.

1. Provost, 2003, p. 630-631. Dans cet article, Provost retrace les différents paradigmes autour de la complexité et de la simplicité en musique jusqu'à l'époque contemporaine. II constate en conclusion de l'article que les débats plutôt théoriques qui se sont imposés au $x x^{e}$ siècle se sont éclipsés au profit d'une "profonde recherche d'identité", la musique ayant cette capacité de transcender l'écriture et la "complexité conceptuelle" qu'elle a su imposer à travers I'histoire.

2. Les citations de Katia Makdissi-Warren sons issues d'entrevues avec l'autrice en octobre 2018 et en juin 2019.

3. Oktoécho, Saimaniq, Cross Current Music, CCM wS2004, 2017.

4. Voir le "Glossaire raisonné" de Bhagwati, 2018, p. 15.

5. “Dans notre société québécoise composée en grande partie d'immigrants, inscrire une identité culturelle par l'exclusion de tout élément venant de l'extérieur serait tout simplement mal venu ", inscrivait la compositrice dans sa thèse de doctorat, déposée en 2007 à l'Université de Montréal.

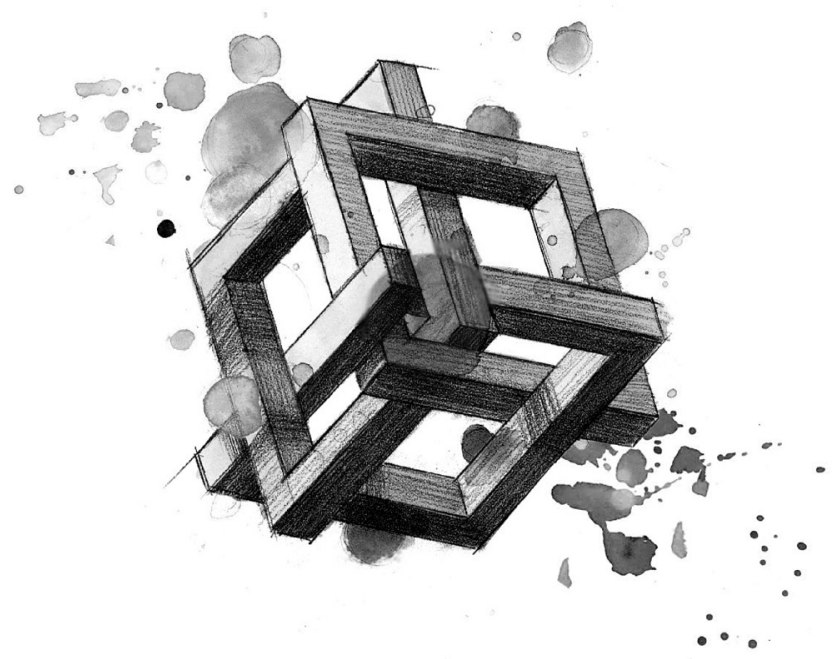

